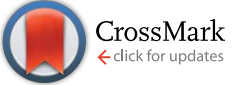

Cite this: RSC Adv., 2017, 7, 11680

Received 17th December 2016 Accepted 5th February 2017

DOI: 10.1039/c6ra28335g

rsc.li/rsc-advances

\title{
Energetics competition in centrally four- coordinated water clusters and Raman spectroscopic signature for hydrogen bonding $\dagger$
}

\author{
Bo Wang, t $^{\text {ab }}$ Wanrun Jiang, ta $^{\text {ab }}$ Yang Gao, ${ }^{\text {ab }}$ Zhiyuan Zhang, ${ }^{\text {ab }}$ Changqing Sun, ${ }^{c}$ \\ Fang Liu*ab and Zhigang Wang*ab
}

Extending the electronic structure of four-coordinated hydrogen bonds ( $\mathrm{H}$-bonds) to medium sized water cages $\left(\mathrm{H}_{2} \mathrm{O}\right)_{n}(n=17,19,20,21,23$ and 25), we separate the $\mathrm{H}$-bonded neighbour molecules of their centrally four-coordinated water (C4CW) molecules from other molecules in outer cages and discover these two regions interact competitively with the central molecule, showing complementary interaction energy curves with respect to size changes. Raman spectral analyses clearly reveal the characteristic vibration response of water molecules to different $\mathrm{H}$-bonding environment, where the $\mathrm{C} 4 \mathrm{CW}$ structure is relatively sensitive. Our theoretical research advances a new perspective for the study of $\mathrm{H}$-bonding interaction in liquid water.

\section{Introduction}

The hydrogen bond (H-bond) plays a significant role in physics, chemistry, biology and environmental sciences. ${ }^{1-5}$ In general, each water molecule within the static liquid water model is bonded to its four nearest neighbor water molecules via two donor and two acceptor bonds, to form the centrally fourcoordinated water (C4CW) structure. Two kinds of $\mathrm{H}$-bonds, strong and weak, exist in liquid water, ${ }^{6,7}$ which is a key factor for determining the properties of water. ${ }^{8}$ The exchange of strong and weak $\mathrm{H}$-bonds enhances the competition between the open and collapsed structures. ${ }^{7}$ For these reasons, a great number of detailed spectroscopic and energetic studies have been carried out for C4CW structures. ${ }^{9-12}$ Theoretical studies ${ }^{9,13}$ have revealed the interactive strength of the $\mathrm{C} 4 \mathrm{CW}$ molecule under the ambient conditions. In particular, some recent experimental and theoretical reports have revealed the covalent-like properties of the H-bond, ${ }^{\mathbf{8}, \mathbf{1 4}-\mathbf{1 6}}$ which further implies the interaction complexity in water clusters through the brought electronic structure anisotropy. In fact, notable differences may exists when comparing the interaction between the $\mathrm{C} 4 \mathrm{CW}$ molecule and its nearest neighbor four water (NN4W) molecules with that

\footnotetext{
${ }^{a}$ Institute of Atomic and Molecular Physics, Jilin University, Changchun 130012, China. E-mail: fliu110@163.com; wangzg@jlu.edu.cn

bilin Provincial Key Laboratory of Applied Atomic and Molecular Spectroscopy, Jilin University, Changchun 130012, China

${ }^{c}$ School of Electrical and Electronic Engineering, Nanyang Technological University, Singapore 639798, Singapore

$\dagger$ Electronic supplementary information (ESI) available. See DOI: $10.1039 / \mathrm{c} 6 \mathrm{ra} 28335 \mathrm{~g}$

\$ These authors contributed equally to this work.
}

between the C4CW molecule and surface molecules of water cages (hereafter referred to as rest water molecules). Therefore, the aim of this work is to demonstrate the energetics relation between the C4CW molecule and other water molecules in medium-sized clusters, so that to help connecting the fundamental four-coordinated hydrogen bond model with the behavior of liquid water under ambient condition.

Water clusters are elementary functional units of liquid water meanwhile are used as ideal theoretical models for investigating the properties of H-bonds. Various reports have already indicated that the most stable structures of water clusters $\left(\mathrm{H}_{2} \mathrm{O}\right)_{n}(n=3-5)$ are cyclic structures ${ }^{17-22}$ and a transition from two-dimensional to three-dimensional structures appears at $n=6 .^{23-25}$ For $n>6$, the minimum energy structures are threedimensional, with all molecules constructing the surface of the cluster. ${ }^{22,26,27}$ For $n \geq 17$, stable structures with odd-numbered molecules are $\mathrm{C} 4 \mathrm{CW}$ clusters ${ }^{\mathbf{9}, 28,29}$ and the recent experimental observations show that the C4CW cluster is also stable for $n=$ $20 .^{30}$ Consequently, C4CW clusters provide a suitable platform for investigating the medium sized water clusters which is capable to capture the nature of liquid water through providing $\mathrm{H}$-bonds in obviously relatively complex coordination environments.

It is important to understand the nature of liquid water. The covalent-like characteristics of $\mathrm{H}$-bonds between water molecules prompt us to explore the interaction mechanism in water clusters from the perspective of an electronic-structure based division of their geometrical structures. Here, we report a theoretical study of C4CW clusters $\left(\mathrm{H}_{2} \mathrm{O}\right)_{n}(n=17,19,20,21,23$ and 25 ), based on the hierarchical models, which divide every $\mathrm{C} 4 \mathrm{CW}$ cluster into three parts: a C4CW molecule, NN4W molecules and rest water molecules. Apparently, the same level of 
calculation is helpful in obtaining accurate results for the three parts. So geometry optimizations employed the empirical dispersion-corrected density functional theory (DFT-D3) method with hybrid generalized gradient-approximation (hybrid GGA) at PBE0-D3/6-31+G(d, p) level. ${ }^{31-34}$ To confirm the obtained structures are truly energy minimum, vibrational frequency verifications have also been carried out at the same level of theory. Furthermore, isotopic substitution was calculated to explore Raman spectra in water clusters. The calculations are performed in the Gaussian 09 program. ${ }^{35}$

\section{Results and discussion}

First of all, we show the stable structures of $\mathrm{C} 4 \mathrm{CW}$ clusters in Fig. 1. Moreover, the Cartesian coordinates for all optimized structures provided are in part 1 of the (ESI $\dagger)$. It can be seen that the $\mathrm{C} 4 \mathrm{CW}$ molecule for each structure is bonded to NN4W molecules via two donor and two acceptor bonds. To verify the reliability of the corresponding region-divided models, we analyzed the electron density difference taking the $\mathrm{C} 4 \mathrm{CW}$ molecule and the whole outer water cage as two interacting monomers. The electron density difference was calculated using the following formula, $\rho=\rho_{\text {total }}-\rho_{\mathrm{C} 4 \mathrm{CW}}$, where $\rho_{\text {total }}$ is the electron density of the whole water cluster and $\rho_{\mathrm{C} 4 \mathrm{CW}}$ is the electron density of $\mathrm{C} 4 \mathrm{CW}$ molecule. The results show that electron accumulation and depletion mainly appears between the C4CW molecule and NN4W molecules, without exception. This indicates the NN4W molecules dominate the electronic structure response in the interaction between the center molecule and the outer cage. Dividing NN4W molecules form the rest part of the cage could be reasonable and may provide insights for understanding the total interaction.

The interaction between the C4CW molecule and its outer water cage could be different due to the adding of water

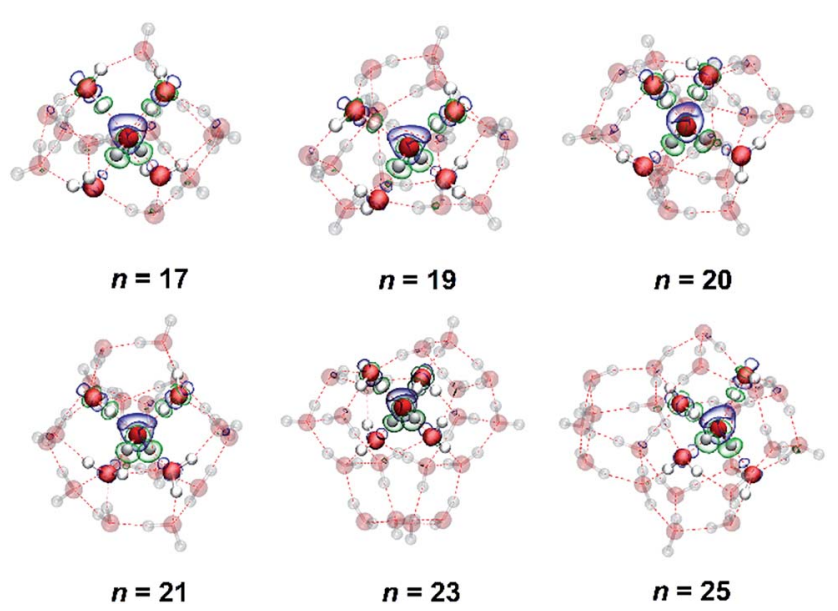

Fig. 1 Electron density difference of $\mathrm{C} 4 \mathrm{CW}$-centered $\left(\mathrm{H}_{2} \mathrm{O}\right)_{n}$ clusters ( $n=17,19,20,21,23$ and 25). Oxygen atoms appear in red, hydrogen in white and the broken red lines denote the $\mathrm{O}: \mathrm{H}$ nonbonds. The $\mathrm{C} 4 \mathrm{CW}$ molecule with its NN4W molecules are drawn in solid atoms while the rest molecules in outer cage are transparent. The blue and green regions represent the electron accumulation and depletion, respectively. molecules in the cage. Hence, the interaction energies between the $\mathrm{C} 4 \mathrm{CW}$ molecule and the overall water cage $\left(E_{\mathrm{int}}\right)$, between the $\mathrm{C} 4 \mathrm{CW}$ molecule and its NN4W molecules $\left(E_{\text {int(C-4) }}\right)$ and between the $\mathrm{C} 4 \mathrm{CW}$ molecule and the rest water molecules $\left(E_{\text {int(C-rest })}\right)$ were respectively calculated and compared among different clusters (see Fig. 2, the details are provided in part 2 of the ESI $\dagger$ ). The results have been corrected for basis set superposition error through the counterpoise correction. Fig. 2a shows that the interaction represented by $E_{\text {int(C- } 4)}$ is always stronger than that by $E_{\text {int(C-rest) }}$ for different cluster size $n$, and a stronger $E_{\mathrm{int}(\mathrm{C}-4)}$ should correspond to a weaker $E_{\text {int(C-rest) }}$, indicating two parts of the water cage always interacts competitively with the $\mathrm{C} 4 \mathrm{CW}$ molecule. Furthermore, another common rule is noteworthy that, two curves of $E_{\text {int }}$ and $E_{\text {int(C-4) }}$ show the same trend thus $E_{\text {int }}$ is dominated by the $E_{\text {int(C-4) }}$, which is in consistent with the previous implication of electronic structure response results in Fig. 1. In addition, we also calculated the average interaction energy per water molecule $\left(E_{2}\right.$, black line in Fig. 2b) and the total number of H-bonds for these $\mathrm{C} 4 \mathrm{CW}$ clusters (blue line in Fig. 2b). The results show that with the increasing $n$, the absolute value of $E_{2}$ and the total number of $\mathrm{H}$-bonds generally increase, while the $E_{2}$ deviation of this trend for $n=20$ and 23 may be attributed to the complexity of geometric structures.
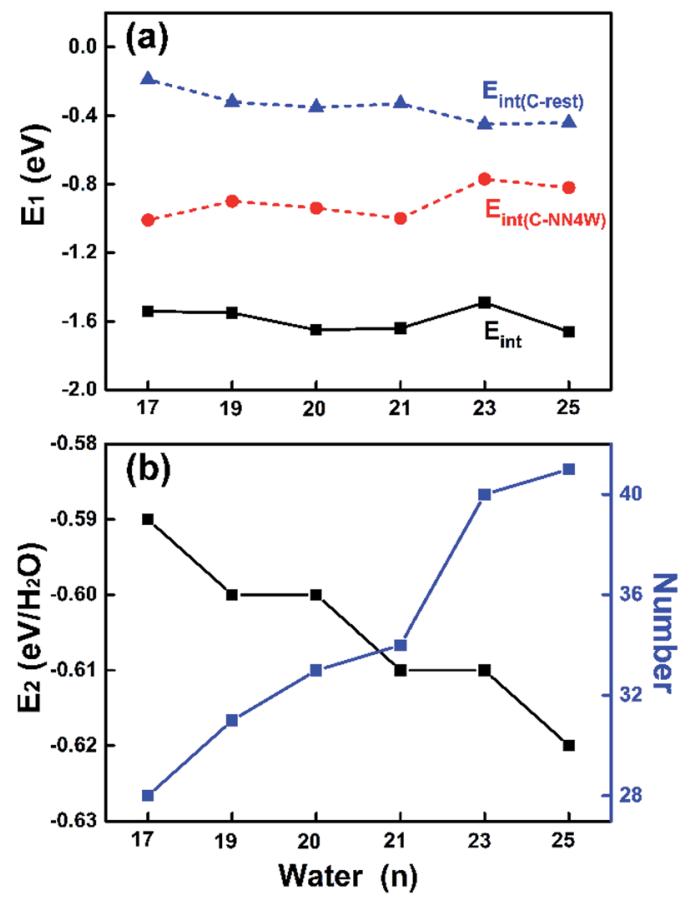

Fig. 2 (a) Total interaction energy of the $\mathrm{C} 4 \mathrm{CW}$ molecule with its

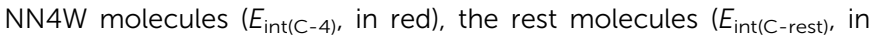
blue) and the overall water cage ( $E_{\text {int, }}$ in black). (b) Average interaction energy per water molecule ( $E_{2}$, in black) of the $C 4 C W$ cluster and the total number of $\mathrm{H}$-bonds (in blue). In detail, $E_{\text {int }}=E_{\text {total }}-E_{\mathrm{C}}-E_{\text {cage, }}$ $E_{\text {int(C-NN4W) }}=E_{\mathrm{C}-\mathrm{NN} 4 \mathrm{~W}}-E_{\mathrm{C}}-E_{\mathrm{NN} 4 \mathrm{~W}}$ and $E_{\text {int(C-rest) }}=E_{\mathrm{C} \text {-rest }}-E_{\mathrm{C}}-$ $E_{\text {rest, }}$ where $E_{\text {total }}, E_{\mathrm{C}-\mathrm{NN} 4 \mathrm{~W}}$ and $E_{\mathrm{C} \text {-rest }}$ is respectively the total energy of all molecules, the C4CW molecule with its NN4W molecules and the C4CW molecule with the rest outer cage molecules. $E_{\mathrm{C}}, E_{\mathrm{NN} 4 \mathrm{~W}}$ and $E_{\text {cage }}$ is the total energy of the C4CW molecule, the NN4W molecules and all molecules in the outer cage, respectively. 
To further illustrate the interaction behavior in mediumsized water clusters and to possibly facilitate experimental structure recognitions through spectroscopic means, we calculated and analyzed Raman spectra for these six clusters. The characteristic range for stretching vibrations of $\mathrm{O}-\mathrm{H}$ covalent bonds are chosen. This region reported as a signature is the focused on previous studies. ${ }^{36,37}$ These $\mathrm{O}-\mathrm{H}$ bonds are classified into different groups according to their intermolecular H-bonding environments. Correspondingly, 4 distinguish parts appear from the total spectra in Fig. 3 (the mass-weighted atomic displacements of these $\mathrm{O}-\mathrm{H}$ stretching modes for $n=17$ ). The part $\mathrm{A}$ is from those $\mathrm{H}$-bonded ones whose oxygen atoms possess the other $\mathrm{H}$ atom dangling outside the cluster, which can be found around some vertexes of the cluster. The part B is from those belonging to the $\mathrm{C} 4 \mathrm{CW}$ molecule and have involved in its $\mathrm{H}$-bonding four-coordination. The part $\mathrm{C}$ is from those in undercoordinated water molecules without dangling $\mathrm{O}-\mathrm{H}$ groups, which can be found around some concave regions of the cluster. The sharp part D is from those dangling ones. At last, there are still some residual peaks in the total spectra curve in Fig. 3. Shown in this implicit way, these are the fifth contribution from $\mathrm{O}-\mathrm{H}$ covalent bonds in NN4W molecules, given NN4W molecules satisfy all exception conditions above as no dangling groups, not at the center and not undercoordinated. In addition, isotopic substitution is employed to investigate Raman spectra. This technique has been widely used in spectroscopy studies. ${ }^{38,39}$ We studied two isotopic mixtures, one is deuterated rest of the water molecules apart (see Fig. S1 $\dagger$ ), the other is deuterated C4CW molecule (see Fig. S2 $\dagger$ ). A comparison of the deuterated vs. undeuterated systems showed practically the same characteristic vibration response in the Raman spectra. These results provide further support to the hierarchical models.

It is shown that, O-H stretching Raman differences among six water clusters are most clearly reflected by the shift and

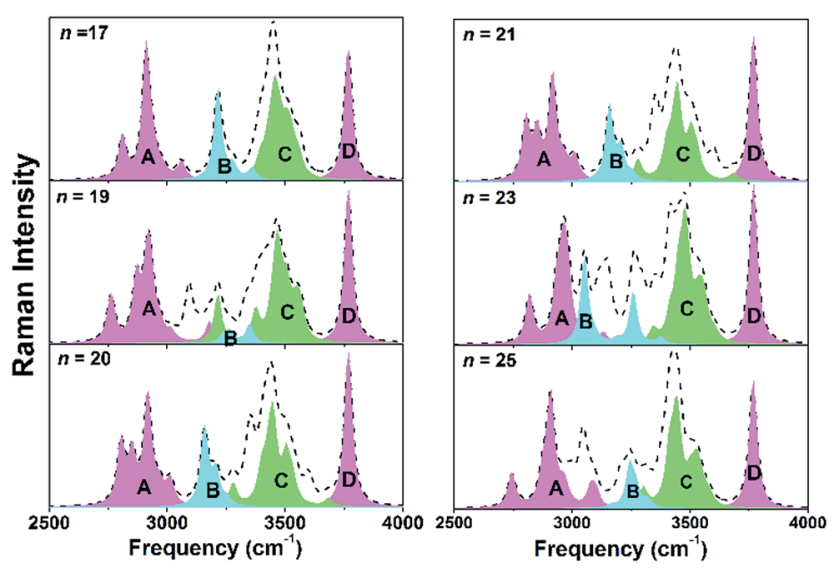

Fig. 3 Raman spectra for $\mathrm{O}-\mathrm{H}$ bond stretching vibrations in $\mathrm{C} 4 \mathrm{CW}$ water clusters $\left(\mathrm{H}_{2} \mathrm{O}\right)_{n}(n=17,19,20,21,23$ and 25$)$ (scaled by 0.9547). The black dashed lines show features from all bonds in entire clusters. Part $\mathrm{A}$ is from those $\mathrm{H}$-bonded ones whose oxygen atoms possess the other $\mathrm{H}$ atom dangling outside the cluster. Part $\mathrm{B}$ is from bonds belonging to the $\mathrm{C} 4 \mathrm{CW}$ molecule. Part $\mathrm{C}$ is from those in the undercoordinated water molecules without dangling $\mathrm{O}-\mathrm{H}$ groups and the sharp part $D$ corresponds to the dangling ones. deformation of the C4CW part B and NN4W residual peaks. Comparisons between $n=19$ and 20 as well as between $n=21$ and 23 provide two most typical cases for this phenomenon. It implies the vibration response of $\mathrm{C} 4 \mathrm{CW}$ structures might be able to characterize the size revolution of medium-sized water clusters. Besides, the synergy between the spectral response of part B and NN4W signals also indicates the C4CW molecule interacts with the NN4W molecules relatively strong so that two regions could influence each other through their H-bonding. This is in consistent with above energetic and electronic structure analyses and further supports our region-divided model. On the other hand, part A, C and D are less sensitive to the size changes. Part $\mathrm{D}$ almost show no notable response since these $\mathrm{O}-\mathrm{H}$ dangling bonds are hardly influenced by the surrounding intermolecular environment. Similar stiffen effects for such stiffer frequencies have also been observed in other water systems and are attributed to the undercooridnation..$^{\mathbf{4 0 4 1}}$ Reasonably, a common feature of parts A, C and D is corresponding $\mathrm{O}-\mathrm{H}$ bonds are all in undercoordinated water molecules.

\section{Conclusions}

In conclusion, this study gives an access to the quantitative analysis on interaction nature of $\mathrm{C} 4 \mathrm{CW}$ clusters, from the perspective of electronic-structure based region-divided models. The energetics results demonstrate the NN4W molecules and the rest outer-cage water molecules interact competitively with the C4CW molecule in medium-sized water clusters. $\mathrm{O}-\mathrm{H}$ bond Raman spectra present distinguish features with the respect to different $\mathrm{H}$-bonding environments and indicate the $\mathrm{O}-\mathrm{H}$ bonds of the $\mathrm{C} 4 \mathrm{CW}$ water molecules and their NN4W molecules have size-sensitive vibrational frequencies. Besides, further study on the dynamic simulation ${ }^{\mathbf{4 2}}$ and methodology ${ }^{\mathbf{4 3}}$ of water clusters are also the direction of our efforts. Hence, we hope that our findings could promote the understanding of the interaction nature towards the liquid water and help to predict the complex behavior of this most ubiquitous substance.

\section{Acknowledgements}

The work was supported by the National Natural Science Foundation of China (11374004 and 11674123). Z. W. also acknowledges the Fok Ying Tung Education Foundation (142001) and High Performance Computing Center of Jilin University.

\section{Notes and references}

1 G. C. Pimentel and A. L. McClellan, The Hydrogen Bond, W. H. Freeman, San Francisco, 1960.

2 S. Scheiner, Hydrogen Bonding: A Theoretical Perspective, Oxford University Press, New York, 1997.

3 G. A. Jeffrey and W. Saenger, Hydrogen Bonding in Biological Structures, Springer-Verlag, Berlin, 1994.

4 G. Gilli and P. Gilli, The Nature of the Hydrogen Bond: Outline of a Comprehensive Hydrogen Bond Theory, Oxford University Press, New York, 2009. 
5 C. Q. Sun and Y. Sun, The Attribute of Water, Springer, Singapore, 2016.

6 J. C. Li and A. I. Kolesnikov, J. Mol. Liq., 2002, 100, 1.

7 Y. Tu and H. Fang, Phys. Rev. E: Stat., Nonlinear, Soft Matter Phys., 2009, 79, 016707.

8 H. Elgabarty, R. Z. Khaliullin and T. D. Kuhne, Nat. Commun., 2015, 6, 8318.

9 J. P. Furtado, A. P. Rahalkar, S. Shanker, P. Bandyopadhyay and S. R. Gadre, J. Phys. Chem. Lett., 2012, 3, 2253.

10 T. D. Kuhne and R. Z. Khaliullin, Nat. Commun., 2013, 4, 1450.

11 T. Hamashima, K. Mizus and A. Fujii, J. Phys. Chem. A, 2011, 115, 620.

12 A. Hermann, W. G. Schmidt and P. Schwerdtfeger, Phys. Rev. Lett., 2008, 100, 207403.

13 F. F. Wang, G. Jenness, W. A. Al-Saidi and K. D. Jordan, J. Chem. Phys., 2010, 132, 134303.

14 J. Zhang, P. Chen, B. Yuan, W. Ji, Z. Cheng and X. Qiu, Science, 2013, 342, 611.

15 B. Wang, W. Jiang, Y. Gao, B. K. Teo and Z. Wang, Nano Res., 2016, 9, 2782.

16 B. Wang, W. Jiang, X. Dai, Y. Gao, Z. Wang and R. Q. Zhang, Sci. Rep., 2016, 6, 22099.

17 M. B. Day, K. N. Kirschner and G. C. Shields, J. Phys. Chem. A, 2005, 109, 6773.

18 K. Kiu, M. G. Brown, J. D. Cruzan and R. J. Saykally, Science, 1996, 271, 62.

19 N. Pugliano and R. J. Saykally, Science, 1992, 257, 1937.

20 J. C. Howard and G. S. Tschumper, J. Chem. Theory Comput., 2015, 11, 2126.

21 R. J. Saykally and D. J. Wales, Science, 2012, 336, 814.

22 P. Qian, W. Song, L. Lu and Z. Yang, Int. J. Quantum Chem., 2010, 110, 1923.

23 M. Losada and S. Leutwyler, J. Chem. Phys., 2002, 117, 2003.

24 Y. Wang, V. Babin, J. M. Bowman and F. Paesani, J. Am. Chem. Soc., 2012, 134, 11116.

25 J. J. I. Foley and D. A. Mazziotti, J. Phys. Chem. A, 2013, 117, 6712.
26 H. M. Lee, H. M. S. B. Suh, J. Y. Lee, P. Tarakeshwar and K. S. Kim, J. Chem. Phys., 2000, 112, 9759.

27 S. Bulusu, S. Yoo, E. Apra, S. Xantheas and X. C. Zeng, J. Phys. Chem. A, 2006, 110, 11781.

28 S. Yoo, E. Apra, X. C. Zeng and S. S. Xantheas, J. Phys. Chem. Lett., 2010, 1, 3122.

29 J. Cui, H. Liu and K. D. Jordan, J. Phys. Chem. B, 2006, 110, 18872.

30 C. C. Pradzynski, C. W. Dierking, F. Zurheide, R. M. Forck, U. Buck, T. Zeuch and S. S. Xantheas, Phys. Chem. Chem. Phys., 2014, 16, 26691.

31 S. Grimme, J. Antony, S. Ehrlich and H. Krieg, J. Chem. Phys., 2010, 132, 154104.

32 J. P. Perdew, K. Burke and M. Ernzerhof, Phys. Rev. Lett., 1996, 77, 3865.

33 C. Adamo and V. Barone, J. Chem. Phys., 1999, 110, 6158.

34 J. P. Perdew, K. Burke and M. Ernzerhof, Phys. Rev. Lett., 1997, 78, 1396.

35 M. J. Frisch, G. W. Trucks, H. B. Schlegel, G. E. Scuseria, M. A. Robb, J. R. Cheeseman, et al., Gaussian 09, Revision D.01, Gaussian, Inc., Wallingford, CT, 2009.

36 Q. Du, E. Freysz and Y. R. Shen, Science, 1994, 264, 826.

37 C. S. Tian and Y. R. Shen, Proc. Natl. Acad. Sci. U. S. A., 2009, 106, 15148.

38 C. S. Tian and Y. R. Shen, J. Am. Chem. Soc., 2009, 131, 2790. 39 M. Shao, J. Keum, J. Chen, Y. He, W. Chen, J. F. Browning, J. Jakowski, B. G. Sumpter, I. N. Ivanov, Y. Z. Ma, C. M. Rouleau, S. C. Smith, D. B. Geohegan, K. Hong and K. Xiao, Nat. Commun., 2014, 5, 3180.

40 U. Buck and F. Huisken, Chem. Rev., 2000, 100, 3863.

41 K. E. Otto, Z. Xue, P. Zielke and M. A. Suhm, Phys. Chem. Chem. Phys., 2014, 16, 9849.

42 V. Ravi Kumar, C. Verma and S. Umapathy, J. Chem. Phys., 2016, 144, 064302.

43 M. J. Gillan, D. Alfè and A. Michaelides, J. Chem. Phys., 2016, 144, 130901. 